\title{
Towards a three-dimensional geological model of the North Sea subsurface
}

\author{
Marcel Bakker ${ }^{1}$, Sjef Meekes ${ }^{2}$, Sytze van Heteren ${ }^{1}$, Denise Maljers ${ }^{1}$, Bob Paap ${ }^{3}$, Simon Fitch ${ }^{4}$, Vincent \\ Gaffney $^{4}$ and Ben Gearey ${ }^{4}$
}

${ }^{1}$ TNO Geological Survey of the Netherlands, PO Box 80015, NL-3508 TA Utrecht, The Netherlands, marcel.bakker@tno.nl

${ }^{2}$ TNO Sustainable Geo-Energy, PO Box 80015, NL-3508 TA Utrecht, The Netherlands

${ }^{3}$ Deltares Subsurface and Groundwater Systems, PO Box 85467, NL-3508 AL Utrecht, The Netherlands

${ }^{4}$ IBM Visual and Spatial Technologies Centre, The University of Birmingham, Edgbaston B15 2TT, United Kingdom

\begin{abstract}
The Geological Survey of the Netherlands is extending the 3D model of the Quaternary record as created for the onshore part of the country to the North Sea realm. Onshore, cores are the most important source of information. Offshore, seismic data are at least equally valuable. A recent pilot study has shown that $2 \mathrm{D}$ and $3 \mathrm{D}$ seismic data, originally collected for hydrocarbon exploration, are very useful in delineating much shallower stratigraphic units. This is best achieved by interpreting 3D seismic surveys together with highresolution 2D seismic lines and well data. In the pilot, focus has been on the Oyster Grounds and Silver Well areas in the central part of the Dutch continental shelf. The units modeled thus far include Middle- to Late-Pleistocene strata that have accumulated in the North Sea Basin. The 3D seismic surveys reveal some remarkable, well-preserved sedimentary features that may be difficult to recognize in 2D profiles but are clearly visible in horizontal time slices. Examples include intricate early Holocene tidal-channel systems and striking sets of tunnel valleys formed during multiple glacial episodes. The level of detail provided by the time slices enables paleolandscape scholars to reconstruct environmental settings of Mesolithic (mostly Holocene) times.
\end{abstract}

\section{INTRODUCTION}

Geological models are quantitative, user-oriented predictions of subsurface architecture and properties in 3D space [Van der Meulen et al., in prep.]. They can be easily queried by end users of geological information to answer questions or make decisions in their respective areas of expertise. Two types of model have been developed for the onshore part of the Netherlands in recent years: (1) layer-based ones, with tops and bases of lithostratigraphic units only, and (2) voxel models composed of a regular grid of 3D pixels (voxels) with associated parameter values. These parameters include lithological characteristics, geotechnical, geochemical and hydrological attributes, and stratigraphy.

Geological modeling has replaced traditional mapping, which focused on producing qualitative visualizations of the subsurface. By using profile-type legends ('Unit X on top of Unit Y on top of Unit Z'), maps depicting the shallow subsurface provide some information on the variability of sedimentary successions, but digital geological models are much better able to show this variability. Although the maps have considerable detail, application possibilities are limited because they are not tailormade for specific purposes. They have been used mainly for illustrative purposes, and have contributed significantly to our understanding of process-response relationships and to the development of geological concepts, including aspects of preservation.

After a successful transition onshore, resulting in the models DGM, NL3D and GeoTOP developed by the Geological Survey of the Netherlands, offshore geological mapping is now being

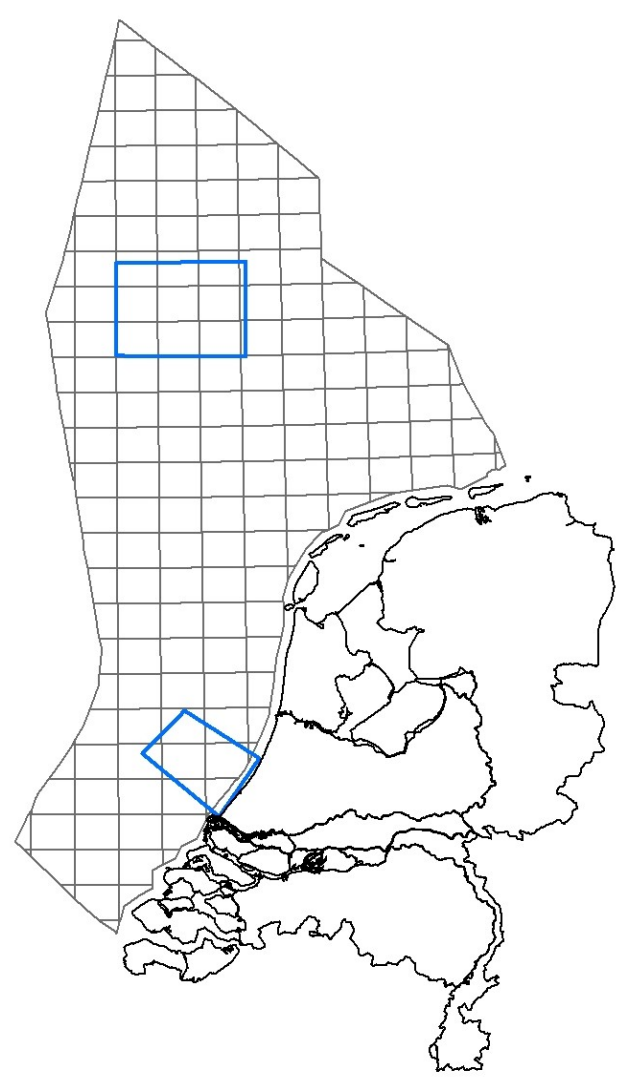

Figure 1. Pilot areas North and South on the Dutch shelf. 
Table 1: Number and penetration depth of boreholes in pilot areas. Number of petroleum-exploration boreholes in parentheses.

\begin{tabular}{ccccc}
\hline Location & Boreholes $0-5 \mathrm{~m}$ & Boreholes $5-12 \mathrm{~m}$ & Boreholes $12-50 \mathrm{~m}$ & Boreholes $>50 \mathrm{~m}$ \\
\hline & & & & \\
Pilot area North & 35 & 39 & 5 & $1(11)$ \\
Pilot area South & 788 & 282 & 111 & $3(34)$ \\
\hline
\end{tabular}

replaced by digital geologic modeling as well. Protocols developed and lessons learned during the development of these onshore models are used as much as possible, but marine mapping and modeling offers its own specific challenges requiring adjustments in approach and methodology. These challenges are related to differences between onshore and offshore data types and data densities. The land data come primarily from boreholes, whereas the offshore has been mapped with a balanced combination of boreholes and geophysical surveys, even for the upper $50 \mathrm{~m}$. Also, the density of boreholes (point data) decreases significantly with increasing distance from the shore (Table 1), forcing us to rely increasingly on 2D (cross-sections) and 3D (full coverage in $\mathrm{x}, \mathrm{y}, \mathrm{z}$ ) seismic data. A second challenge associated with marine mapping is the dynamic nature of this environment. Much more than onshore, sediment is being transported to such an extent that maps of the shallow subsurface may be out of date after as little as a decade.

Here, we report on the development of a protocol for modeling the shallow (upper 100s of m) subsurface of the Dutch part of the North Sea. Efforts to optimize data density are highlighted, as they determine the scale at which lithostratigraphic and lithological units can be recognized and mapped in light of the limited number of boreholes. Using this new protocol, lithological horizons are being digitized to form a layer-based model for two pilot areas (Figure 1), and initial steps are undertaken to build a voxel model that will allow seamless integration with onshore voxel models.

\section{DATA TYPES}

\section{Borehole logs and laboratory analyses}

Borehole logs are extracted from the DINO database developed and hosted by the Geological Survey of the Netherlands. Most information comes from shallow boreholes, typically up to $12 \mathrm{~m}$ deep but locally up to several 100 s of $\mathrm{m}$. The logs of some hydrocarbon-exploration wells provide information for the upper $100 \mathrm{~s}$ of $\mathrm{m}$ as well. Most boreholes up to $5 \mathrm{~m}$ deep provided littledisturbed core samples. The vast majority of deeper boreholes were made with counterflush or related techniques resulting in disturbed samples. Per depth interval, borehole logs provide qualitative, visually assessed information on lithology and stratigraphy. For many boreholes, one or more samples were analyzed in the laboratory, most frequently for quantitative measures of grain-size parameters, micropaleontology or age.

\section{Seismic profiles and time slices}

In marine seismic-reflection surveys, acoustic waves generated by a seismic source towed by or mounted to a vessel are used to obtain information on the subsurface. When a seismic wave encounters a boundary between two materials with different acoustic properties, some of the energy in the wave will be reflected at the boundary, while some of the energy will continue through the boundary. The amplitude of the reflected wave depends on the impedance contrast between the two materials, which is greatest at the water-seabed interface.

A distinction can be made between high-resolution single- or multi-channel systems using frequencies of 100-1000 Hz and lowresolution multi-channel systems using $<100 \mathrm{~Hz}$. High-frequency single-channel systems record through short hydrophones. Their vertical resolution is on the order of a few meters, but they suffer from quality loss below first seabed multiple. High-frequency multi-channel systems record through hydrophone arrays in cables (streamers). Vertical resolution is on the order of meters below $100 \mathrm{~ms}(100 \mathrm{~ms}$ two-way travel time $($ TWTT) $\approx 75-80 \mathrm{~m}$, including the water column), but lower in shallower units. Lowfrequency multi-channel systems record through km-long hydrophone arrays. Vertical resolution is $5-15 \mathrm{~m}$, depending on the frequency, on survey and source characteristics in relation to target geometry and on post-survey processing [Praeg, 2003]. In general, information of the upper $100-200 \mathrm{~ms}$ is lost. When acquisition parameters and processing are optimized for the shallow subsurface, however, information may be obtained from the seabed downward (starting at about $50 \mathrm{~ms}$ ) when water depth is at least $40 \mathrm{~m}$. In 3D surveys, multiple streamers are deployed in parallel to record data suitable for $3 \mathrm{D}$ interpretation of the structures beneath the seabed. Most 3D surveys employ lowfrequency systems, which work best in deeper waters and cover very large areas efficiently at a coarse resolution [Fitch et al., 2005].

Data collected via a single cable or streamer are displayed as vertical cross-sections through the subsurface. In our traditional surveys conducted for mapping purposes, distances between adjacent profiles are typically on the order of $10 \mathrm{~km}$ or more. Such a wide spacing is commonly sufficient to understand the overall architecture and formation of sedimentary systems, and may be good enough for pre-planning purposes, but render maps unsuitable for most applied use. Not all reflections can be correlated among lines, and most architectural elements have lateral dimensions smaller than can be resolved in a $10 \times 10 \mathrm{~km}$ grid.

Data collected with multiple parallel streamers may be displayed as vertical cross sections, time slices or horizon slices. Time slices are sections of 3D seismic data having a certain arrival time. Because of spatial variability of sound velocity in the subsurface, they are near-horizontal rather than perfectly horizontal depth slices. Because of their map view, time slices are very suitable to identification of landscape elements as reflected in seismic amplitude. Horizon slices show the spatial pattern of particular reflections, created by tracing these reflections on all survey lines and interpolating the resulting data. Thus, features 
can be extracted not only in planform but also in $3 \mathrm{D}$, providing depth (and relative age) relationships.

\section{WORKFLOW}

\section{Stratigraphic framework}

The stratigraphic framework for the pilot studies is provided by published 1:250,000 map sheets made jointly by the British, Belgian and Dutch geological surveys during the 1980s and 1990s, and by the lithostratigraphic overview of Rijsdijk et al. [2005]. The borehole and seismic data are used to identify and map the tops of stratigraphic units. In identifying these units, welldefined reflections of 2D and 3D seismic datasets are linked and labeled systematically from the mid-Miocene unconformity upward, focusing on vertical (TWTT) mismatches at line intersections. These mismatches relate in part to positioning inaccuracies of older surveys.

\section{Petrel project}

At the core of the interpretation activities, a mother file was created in Petrel, a software application for the visualization and analysis of aggregated reservoir data from multiple sources. This mother file hosts all interpreted horizons. For interpretation, elements of the mother file are copied onto local hard drives. Changes and additions made on such local copies are exported regularly to the mother file.

Although the 3D seismic data allow superior visualization of even small sedimentary units, they are not ideally suited for the development of an overall stratigraphic framework of shallow $(<300 \mathrm{~m})$ units in Petrel. Therefore, we started reinterpreting highquality 2D seismic lines collected during the 1980s and 1990s for the 1:250,000 maps. These lines were labeled, linking the major reflections to boundaries between stratigraphic units, and providing metadata including certainty of interpretation and name of interpreting geologist. The labeling scheme (for both bounding reflections and seismic facies) is summarized and updated in a table that includes information on reflection strength, internal reflection configuration, external form of bounded unit, degree of transparency and position relative to the reference datum (mean sea level).

The resulting framework is validated and supplemented with $3 \mathrm{D}$ seismics. By scrolling up and down in 3D seismic cubes at intersection planes with 2D seismic profiles, seismic facies and reflections identified on the $2 \mathrm{D}$ data can partly be assigned to depositional settings and environments. More subtle features on the $2 \mathrm{D}$ lines that were missed initially are labeled as part of this validation step. Finally, the 3D data are interpreted in their own right.

The seismic interpretations are validated with borehole data and with the results of laboratory analyses.

\section{Export to modeling software (ISATIS)}

The horizon slices as reconstructed in Petrel are exported as $\mathrm{x}$ $y$-t grids with a time rather than a depth value for each $x-y$ coordinate. The $\mathrm{t}$ values are translated into $\mathrm{z}$ values using seismicwave velocities of $\mathrm{x} \mathrm{ms}^{-1}$. The resulting output is imported into Isatis, geostatistical software used to generate a digital geological model.

\section{WORKFLOW OUTPUT}

\section{Pilot area North}

The northern pilot area offers high-quality 3D seismics supplemented with 2D seismics and few boreholes. Using the workflow developed as part of the pilot study, we reconstructed the top of the Lower-Pleistocene Markams Hole Formation for an area of $55 \times 65 \mathrm{~km}$ (Figure 2). When viewed at this large scale, this horizon slice provides a general impression of highs and lows, with an overall deepening from the southwest to the northeast. The eastern half of the image shows more complicated patterns associated with faults.

When zooming in and intersecting seismic profiles or horizon slices with time slices, sub-kilometer-scale patterns visible on the time slices provide direct indications of depositional environments. Some features can be easily recognized and interpreted, such as tunnel valleys, iceberg-scour marks and various channel fills. Tunnel valleys are large, valleys formed by meltwater under Pleistocene ice sheets near their margins. They may be up to $100 \mathrm{~km}$ long, kilometers wide and $100 \mathrm{~s}$ of meters deep. The scour marks of icebergs, formed in open-marine waters beyond ice-sheet margins, appear as thin lines, commonly with a fairly uniform direction. Channel fills show intricate meandering and dendritic patterns (Figure 3). They may be tidal or fluvial in origin, and Pleistocene or Holocene in age.

The clear visibility of some of the smaller features on time slices is most likely related to the presence of shallow gas [cf. Schroot and Schüttenhelm, 2003], either formed in situ (e.g., organics in tidal-channel fills) or supplied from an external source.

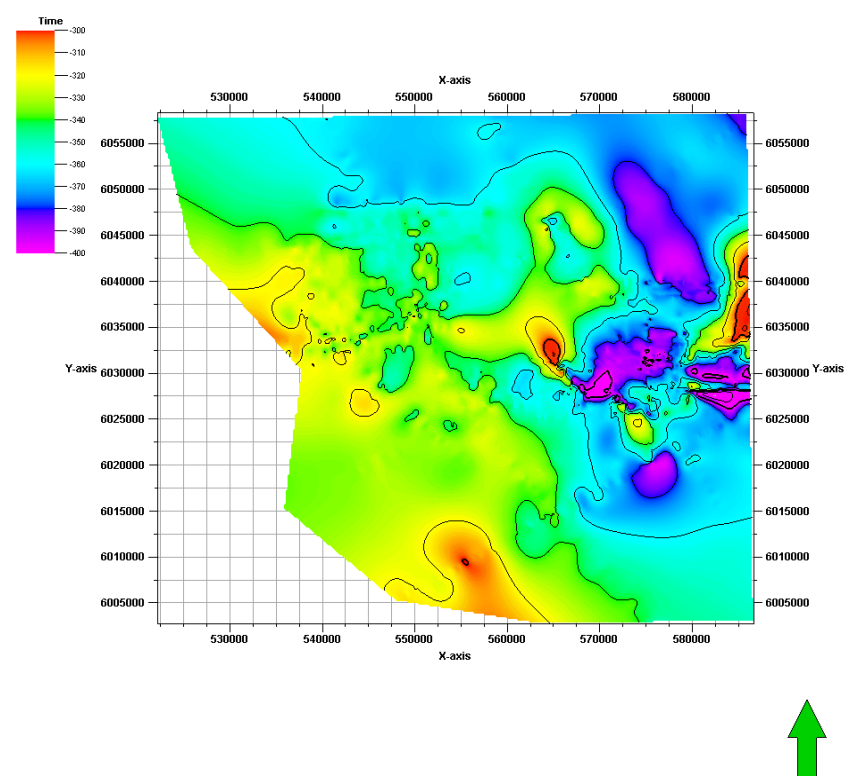

Figure 2. Horizon slice of Markams Hole Formation in pilot area South. Darker graytones denote lows. 


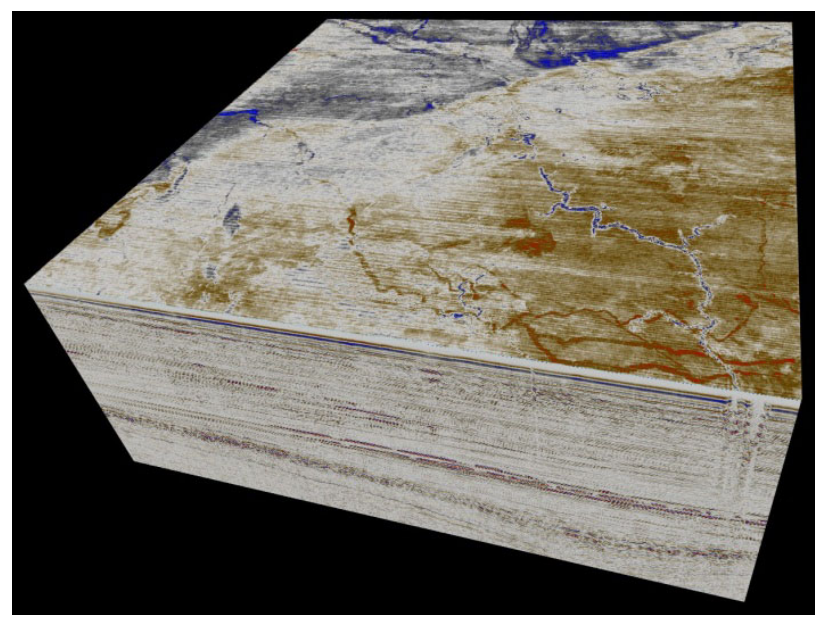

Figure 3. 3D seismic cube of a $20 \times 20 \mathrm{~km}$ area with early Holocene tidal-channel systems in the time slice.

\section{Pilot area South}

The southern pilot area offers low-quality 3D seismics supplemented with 2D seismics and abundant boreholes. The low quality of the 3D seismics for the upper subsurface units in these shallower waters is related to system settings targeted at optimizing data quality for the deeper, petroleum-bearing units, and to a paucity of sediments containing shallow gas. Using the workflow developed as part of the pilot study, we were able to identify the remains of a once near-continuous horizon of Basal Peat covering the Pleistocene landscape that drowned during the early Holocene.

Validation with borehole data shows that the Basal Peat imaged on the time slices of the 3D seismics is very thin and buried under no more than a few $\mathrm{m}$ of clastic sediment. The fact that very shallow, decimeter-thick organic units can be identified and mapped using acoustic methods with a vertical resolution of 5-15 $\mathrm{m}$ shows the possibilities of our new approach. A disadvantage is the persistence of the gas-related reflections. Although the Basal Peat is very thin, its apparent thickness as derived from subsequent time slices is on the order of $10 \mathrm{~s}$ of meters. When borehole data are scarce, it may be difficult to constrain the real thickness of imaged units.

\section{DISCUSSION}

From the pilot study, it has become apparent that the joint use of 2D and 3D seismics offers the best chance to extract detailed information, both laterally and vertically, from acoustic datasets. Time slices from 3D seismics are useful for identifying familiar sedimentary features such as channel fills and deltas. They can also be used for an initial validation of non-parallel anomalies on seismic profiles, such as clinoforms and irregular reflections. The temptation to focus on $3 \mathrm{D}$ seismic cubes and ignore $2 \mathrm{D}$ data should be resisted. Sedimentary features that cannot immediately be identified on the basis of their planform are commonly visible on seismic profiles as well, providing additional information on size and shape needed for interpretation. Because of their superiority in vertical resolution, high-frequency $2 \mathrm{D}$ seismics are also a good starting point for selecting and digitizing reflections for which horizon slices are constructed.
Digital geological models that include 3D seismic data are superior in detail to those relying exclusively on 2D seismic data and boreholes. For most end users of geological information, such detail is essential. Marine archeologists, for example, rely heavily on time-slice interpretation for paleolandscape reconstructions [Fitch et al., 2005]. They need to be able to recognize former coastlines, fertile lowlands, and drainage patterns and directions.

The availability of 3D seismics for large parts of the North Sea, related to the abundance and distribution of petroleum reservoirs, provides a unique opportunity to model the shallow subsurface at a spatial resolution comparable to that of the onshore voxel models NL3D $(250 \times 250 \times 1 \mathrm{~m})$ and GeoTOP $(100 \times 100 \times 0.5$ $\mathrm{m})$ and layer-based model DGM $(100 \times 100 \mathrm{~m})$. This similarity in resolution will make it easier to work toward an integrated landsea model of the shallow subsurface. Such a model will help understand subsurface-related processes that are unrelated to the present North Sea shore. Hydrological models, for example, may be optimized when the distribution of units with various hydraulic properties is known both on- and offshore. The creation of a digital geological model for the entire North Sea, and its integration with existing or future onshore models for North Sea countries will be a key step in the development of a webaccessible geo-model for all of Europe.

\section{CONCLUSIONS}

The joint use of $2 \mathrm{D}$ and $3 \mathrm{D}$ seismic data is very useful in delineating shallow stratigraphic units, revealing features that may be difficult to recognize in $2 \mathrm{D}$ profiles. A new workflow is used to delineate unit boundaries and to map features as small as $10 \mathrm{~s}$ of meters laterally and as thin as a decimeter. The presence of shallow gas enhances many of these features but also leads to vastly overestimated apparent thicknesses. The resulting improvement in mapping resolution is a key step toward an integrated land-sea model of the shallow subsurface.

\section{REFERENCES}

Fitch, S., K. Thomson, and V. Gaffney (2005), Late Pleistocene and Holocene depositional systems and the paleogeography of the Dogger Bank, North Sea, Quaternary Research, 64, 185196.

Praeg, D. (2003), Seismic imaging of mid-Pleistocene tunnelvalleys in the North Sea Basin - high resolution from low frequencies, Journal of Applied Geophysics, 53, 273-298.

Rijsdijk, K. F., S. Passchier, H. T. J. Weerts, C. Laban C., R. J. W. van Leeuwen, and J. H. J. Ebbing (2005), Revised Upper Cenozoic stratigraphy of the Dutch sector of the North Sea Basin: towards an integrated lithostratigraphic, seismostratigraphic and allostratigraphic approach, Netherlands Journal of Geosciences, 84(2), 129-146.

Schroot, B. M. and R. T. E. Schüttenhelm (2003), Expressions of shallow gas in the Netherlands North Sea, Netherlands Journal of Geosciences, 82(1), 91-105.

Van der Meulen, M. J., F. S. Busschers, S. H. L. L. Gruijters, J. L. Gunnink, P. Kiden, D. Maljers, J. Stafleu, W. O. van Berkel, M. J. van Bracht, T. M. van Daalen, F. G. van Geer, S. F. van Gessel, S. van Heteren, R. J. van Leeuwen, R. W. Vernes, and W. E. Westerhoff (in prep.), Geological surveying in the Netherlands: trends and perspectives, Netherlands Journal of Geosciences. 